\title{
The sensitivity and specificity of fluorescent leprosy antibody absorption (FLA-ABS) test for detecting subclinical infection with Mycobacterium leprae
}

\author{
JI BAOHONG, ${ }^{*}$ TANG QUANKUI, ${ }^{*}$ LI YENLONG, \\ CHEN JIAKUN, ${ }^{*}$ ZHANG JIALING, ${ }^{*}$ DONG \\ LIWEN, * WANG CHENMIN, $†$ MA JIAJU \\ \& YE DALI§ \\ *Shanghai Zeng Yi Hospital; † Hai-an Leprosy Hospital, Jiangsu \\ Province; $\ddagger$ Hanzhong Sanitorium, Shanxi Province; §Huzhou \\ Epidemic Prevention Station, Zhejiang Province, People's Republic \\ of China
}

\begin{abstract}
Accepted for publication 24 January 1984
Summary From the examination of 854 sera from different sources by the fluorescent leprosy antibody absorption (FLA-ABS) test, the sensitivity and specificity of this test for leprosy has been confirmed. A positive FLA-ABS test in a non-leprosy individual should be considered as an indicator of subclinical leprosy infection. The subclinical infection rates of two endemic areas ranged from 11.4 to $16.3 \%$ and were at least 200 times higher than the cumulative prevalence rate of clinical inf ection. The combination of a positive FLA-ABS test with a negative Mitsuda reaction indicates that the individual has been infected with Mycobacterium leprae, but cell-mediated immunity has not been induced. Such individuals are at a greater risk of developing multibacillary leprosy and should be carefully followed up or some prophylactic measures should be considered. Since subclinical infection cannot be differentiated from very early leprosy by the FLA-ABS test alone, it is not a reliable diagnostic test of early leprosy.
\end{abstract}

\section{Introduction}

A technique for the detection of subclinical infection with Mycobacterium leprae among the population of leprosy endemic areas is one of the most important requirements for leprosy control programmes. The technique should be highly sensitive and specific for leprosy, the procedures and equipments should be simple and convenient for use in the field. Abe et al. ${ }^{1-4}$ have developed the fluorescent 
leprosy antibody absorption (FLA-ABS) test, using the indirect fluorescent antibody technique and improving the specificity by absorption of cross-reacting anti-mycobacterial antibodies in the serum. This test has been claimed to be useful for detecting subclinical leprosy infection. ${ }^{1-6}$ The purpose of the present study is to evaluate the sensitivity and specificity of the FLA-ABS test.

\section{Materials and methods}

SERA

Approximately $0.5 \mathrm{ml}$ serum was collected from each individual and stored at $-20^{\circ} \mathrm{C}$. Sodium azide at final concentration $0 \cdot 1 \%$ was added to those specimens which had to be transported for long distances, these specimens being kept in wet-ice thermos flasks. The sera of leprosy patients were collected from Shanghai Zeng Yi Hospital, Hanzhong Sanitorium (Shanxi Province) and the leprosaria of Suzhou Prefecture (Jiangsu Province). The household contacts of leprosy patients were from Hai-an County (Jiangsu Province), Huzhou (Zhejiang Province) and Chenggu County (Shanxi Province). The healthy inhabitants of leprosy endemic areas were collected from Caoyuan People's Commune of Hai-an County and Lianshi People's Commune of Huzhou. The cumulative leprosy prevalence rates of these two areas were 4.8 and 6.2 per thousand respectively. The doubtful leprosy cases were from the Out-patient Department of Shanghai Zeng Yi Hospital and the professional contacts were the staff of the same hospital. Pulmonary tuberculosis patients were provided by the Shanghai Second Tuberculosis Sanitorium. Sera of healthy non-contacts were obtained from donors in Shanghai.

\section{MYCOBACTERIAL SUSPENSION}

$M$. leprae suspensions were prepared from human leproma by the method of Abe et al. or from $M$. leprae-infected armadillo livers by Protocol 1/79. The latter $M$. leprae were provided by courtesy of Dr R J W Rees, National Institute for Medical Research, London, under the IMMLEP project of WHO. Two forms of armadillo-derived $M$. leprae were obtained: bacillary suspension at a concentration of $10^{10} \mathrm{AFB} / \mathrm{ml}$ and freeze-dried bacilli. The bacillary suspension was diluted to $2 \times 10^{8} \mathrm{AFB} / \mathrm{ml}$ for preparing the smear of $M$. leprae and the results were the same as in those obtained with human-derived $M$. leprae. The freeze-dried bacilli were used for the additional absorption with $M$. leprae. BCG, M. vaccae, $M$. tuberculosis (H37Rv), M. kansasii, M. marinum, M. smegmatis, M. phlei and $M$. avium were cultivated on Ogawa's $1 \%$ egg medium. The colonies were homogenized, washed and suspended in normal saline. 
FLA-ABS TEST

The steps of the test, including cross-reactions with other mycobacteria and additional absorption with $M$. leprae or other mycobacteria, were those described by Abe et al. ${ }^{3,4}$ A fluorescent microscope, Model BHF, Olympus Ltd, Japan, was used throughout this study. The filter system included BV and interference system, Model F-FITC, Olympus Ltd, Japan. The specificity of the green fluorescence as shown by the bacilli under the BV system were confirmed by using the interference system. The criteria for fluorescent microscopic examination were the same as Abe et al. ${ }^{3}$ and two plus or more fluorescence caused by the $1: 40$ or higher dilution of sera were considered to be positive.

\section{Results}

FLA-ABS TEST AMONG LEPROSY PATIENTS

Out of 161 active leprosy patients, $146(90.7 \%)$ were proved positive by the FLA-ABS test. The results of the tests on patients throughout the spectrum of leprosy are shown in Table 1. The positive rate was highest in lepromatous patients and gradually decreased towards the tuberculoid end. However, $73.9 \%$ of TT patients were still positive. The differences in positive rates between LL vs BT or TT, BL vs TT were statistically significant $(P<0.05$ or $P<0.01)$. The distribution of the antibody titres down the spectrum of leprosy showed the same tendency as the positive rate, i.e. the mean antibody titre was highest at the lepromatous end and gradually decreased towards the tuberculoid end. The differences in mean antibody titre between each type of patient, except BL vs BB or BT vs TT, were statistically significant although there were striking variations within each type.

Table 1. FLA-ABS test among active leprosy patients

\begin{tabular}{|c|c|c|c|c|c|c|c|c|c|c|}
\hline \multirow{3}{*}{$\begin{array}{c}\text { Classification } \\
\text { of leprosy }\end{array}$} & \multirow{3}{*}{$\begin{array}{c}\text { No. } \\
\text { of } \\
\text { cases }\end{array}$} & \multirow{2}{*}{\multicolumn{2}{|c|}{ Positive }} & \multicolumn{7}{|c|}{ Distribution of antibody titre $\left(10 \times 4^{x}\right)$} \\
\hline & & & & \multicolumn{5}{|c|}{$x=$} & \multicolumn{2}{|c|}{ Mean } \\
\hline & & No. & $\%$ & 0 & 1 & 2 & 3 & 4 & of $x$ & SD \\
\hline LL & 45 & 45 & 100 & 0 & 3 & 4 & 14 & 24 & $3 \cdot 31$ & $0 \cdot 90$ \\
\hline BL & 50 & 47 & $94 \cdot 0$ & 3 & 9 & 22 & 11 & 5 & $2 \cdot 12$ & $1 \cdot 02$ \\
\hline $\mathrm{BB}$ & 16 & 15 & $93 \cdot 8$ & 1 & 5 & 0 & 7 & 3 & $2 \cdot 38$ & $1 \cdot 31$ \\
\hline BT & 27 & 22 & $81 \cdot 5$ & 5 & 7 & 12 & 2 & 1 & 1.52 & 1.01 \\
\hline TT & 23 & 17 & $73 \cdot 9$ & 6 & 7 & 5 & 4 & 1 & $1 \cdot 44$ & $1 \cdot 20$ \\
\hline Total & 161 & 146 & $90 \cdot 7$ & 15 & 31 & 43 & 38 & 34 & $2 \cdot 28$ & $1 \cdot 26$ \\
\hline
\end{tabular}


Table 2. FLA-ABS test in leprosy patients; grouping by bacterial load or activity of infection

\begin{tabular}{|c|c|c|c|c|c|c|c|c|c|c|c|c|c|}
\hline \multirow{3}{*}{$\begin{array}{c}\text { Classification } \\
\text { of leprosy }\end{array}$} & \multirow[b]{3}{*}{ Group } & \multirow{3}{*}{$\begin{array}{c}\text { No. } \\
\text { of } \\
\text { cases }\end{array}$} & \multirow[b]{3}{*}{ No. } & \multirow{3}{*}{$\begin{array}{l}\text { Positive } \\
\%\end{array}$} & \multicolumn{9}{|c|}{ Distribution of antibody titre $\left(10 \times 4^{x}\right)$} \\
\hline & & & & & & & & $x=$ & & & Mean & & \multirow[b]{2}{*}{$P$} \\
\hline & & & & & $P$ & 0 & 1 & 2 & 3 & 4 & of $x$ & SD & \\
\hline \multirow{2}{*}{ LL } & Smear $(+)$ & 45 & 45 & 100 & - & 0 & 3 & 4 & 14 & 24 & $3 \cdot 31$ & $0 \cdot 90$ & - \\
\hline & Smear $(-)$ & 33 & 30 & $90 \cdot 9$ & $<0.05$ & 3 & 3 & 11 & 8 & 8 & $2 \cdot 46$ & $1 \cdot 23$ & $<0 \cdot 0$ \\
\hline \multirow{2}{*}{ TT } & Active & 23 & 17 & $73 \cdot 9$ & - & 6 & 7 & 5 & 4 & 1 & $1 \cdot 44$ & $1 \cdot 20$ & - \\
\hline & Quiescent & 36 & 12 & $33 \cdot 3$ & $<0.01$ & 24 & 10 & 2 & 0 & 0 & $0 \cdot 39$ & $0 \cdot 60$ & $<0.0$ \\
\hline
\end{tabular}

Table 2 suggested that the results of the FLA-ABS test were well correlated with the bacterial load of the host or the activity of the leprosy infection. The positive rate and mean antibody titre were significantly lower in the smear negative LL group than in those in the smear positive LL group, and the same tendency was observed in the quiescent TT group (where the skin lesions had subsided for at least 3 years) as compared with the active TT group.

\section{FLA-ABS TEST AMONG NON-LEPROSY INDIVIDUALS}

Five hundred and forty-nine non-leprosy cases were examined and the results are summarized in Table 3. All sera from sputum-positive pulmonary tuberculosis patients and healthy non-contacts gave negative results. Among the healthy

Table 3. FLA-ABS test among non-leprosy individuals

\begin{tabular}{|c|c|c|c|c|c|c|c|c|c|c|}
\hline \multirow[b]{3}{*}{ Group } & \multirow{3}{*}{$\begin{array}{c}\text { No. } \\
\text { of } \\
\text { cases }\end{array}$} & \multirow{2}{*}{\multicolumn{2}{|c|}{ Positive }} & \multicolumn{7}{|c|}{ Distribution of antibody titre $\left(10 \times 4^{x}\right)$} \\
\hline & & & & & & $x=$ & & & Mean & \\
\hline & & No. & $\%$ & 0 & 1 & 2 & 3 & 4 & of $x$ & SD \\
\hline Healthy non-contacts & 30 & 0 & 0 & 30 & 0 & 0 & 0 & 0 & 0 & 0 \\
\hline Tuberculosis patients & 30 & 0 & 0 & 30 & 0 & 0 & 0 & 0 & 0 & 0 \\
\hline \multicolumn{11}{|l|}{ Endemic area } \\
\hline Healthy adults (Lianshi) & 147 & 24 & $16 \cdot 3$ & 123 & 21 & 3 & 0 & 0 & $0 \cdot 18$ & 0.43 \\
\hline $\begin{array}{l}\text { Healthy schoolchildren } \\
\text { (Lianshi) }\end{array}$ & 44 & 5 & $11 \cdot 4$ & 39 & 5 & 0 & 0 & 0 & $0 \cdot 11$ & $0 \cdot 32$ \\
\hline $\begin{array}{l}\text { Healthy schoolchildren } \\
\text { (Caoyuan) }\end{array}$ & 117 & 16 & $13 \cdot 7$ & 101 & 13 & 3 & 0 & 0 & $0 \cdot 16$ & $0 \cdot 43$ \\
\hline $\begin{array}{l}\text { Household contacts of } \\
\text { multibacillary leprosy }\end{array}$ & 88 & 58 & $65 \cdot 9$ & 30 & 36 & 17 & 5 & 0 & 0.97 & $0 \cdot 88$ \\
\hline $\begin{array}{l}\text { Household contacts of } \\
\text { paucibacillary leprosy }\end{array}$ & 45 & 8 & $17 \cdot 8$ & 37 & 6 & 2 & 0 & 0 & $0 \cdot 22$ & $0 \cdot 52$ \\
\hline Professional contacts & 48 & 20 & $41 \cdot 7$ & 28 & 8 & 9 & 3 & 0 & $0 \cdot 73$ & 0.98 \\
\hline
\end{tabular}


Table 4. Comparison of FLA-ABS test in professional contacts: grouping by seniority

\begin{tabular}{|c|c|c|c|c|c|c|c|c|c|c|c|c|}
\hline \multirow[b]{3}{*}{ Group } & \multirow{3}{*}{$\begin{array}{l}\text { No. } \\
\text { of } \\
\text { cases }\end{array}$} & \multirow[b]{3}{*}{ No. } & \multirow{2}{*}{\multicolumn{2}{|c|}{ Positive }} & \multicolumn{8}{|c|}{ Distribution of antibody titre $\left(10 \times 4^{x}\right)$} \\
\hline & & & & & & & $x=$ & & & Mean & & \\
\hline & & & $\%$ & $P^{*}$ & 0 & 1 & 2 & 3 & 4 & of $x$ & SD & $P^{*}$ \\
\hline $\begin{array}{l}\text { Seniority } \\
\quad \geqslant 15 \text { years }\end{array}$ & 26 & 19 & $73 \cdot 1$ & - & 7 & 7 & 9 & 3 & 0 & $1 \cdot 31$ & $1 \cdot 01$ & - \\
\hline $\begin{array}{l}\text { Seniority } \\
<5 \text { years }\end{array}$ & 22 & 1 & $4 \cdot 6$ & $<0 \cdot 01$ & 21 & 1 & 0 & 0 & 0 & $0 \cdot 05$ & $0 \cdot 21$ & $<0 \cdot 01$ \\
\hline Total & 48 & 20 & $41 \cdot 7$ & - & 28 & 8 & 9 & 3 & 0 & $0 \cdot 73$ & $0 \cdot 98$ & - \\
\hline
\end{tabular}

* Compare with the group of seniority $\geqslant 15$ years.

inhabitants, excluding the household contacts from the two endemic areas, the positive rates ranged from 11.4 to $16.3 \%$ and the mean antibody titres ranged from $0 \cdot 11$ to $0 \cdot 18 \%$. No significant difference has been observed between the two areas or two age groups from the same area. Both the positive rate and mean antibody titre were very significantly higher in household contacts of multibacillary patients than in those of paucibacillary patients. The so-called professional contacts were the staff working in a leprosy hospital mainly for smear-positive patients. The total positive rate of that group was $41.7 \%$. If subdivided according to seniority in the hospital, the positive rate and mean antibody titre were much higher among the staff with seniority $\geqslant 15$ years than in those with seniority $<5$ years (Table 4).

Mitsuda lepromin $\left(1.6 \times 10^{8} \mathrm{AFB} / \mathrm{ml}\right)$ testing was undertaken immediately after blood collection in 340 healthy inhabitants or household contacts in leprosy endemic areas. The Mitsuda reaction was read at 21-28 days and was considered positive if the average diameter of the nodule was greater than $3 \mathrm{~mm}$. As shown in Table 5, no correlation has been found between the Mitsuda reaction and the FLA-ABS test $\left(\chi^{2}=0 \cdot 264, P>0 \cdot 05\right)$. According to the results of both tests, there were four combinations, i.e. both positive, both negative, Mitsuda positive/

Table 5. Correlation between FLA-ABS test and Mitsuda reaction

\begin{tabular}{llll}
\hline & \multicolumn{2}{c}{ FLA-ABS test } & \\
Mitsuda reaction & Positive & Negative & Total \\
\hline Positive & $34(10 \cdot 0 \%)$ & $152(44 \cdot 7 \%)$ & $186(54 \cdot 7 \%)$ \\
Negative & $19(5 \cdot 6 \%)$ & $135(39 \cdot 7 \%)$ & $154(45 \cdot 3 \%)$ \\
Total & $53(15 \cdot 6 \%)$ & $287(84 \cdot 4 \%)$ & $340(100 \cdot 0 \%)$ \\
\hline
\end{tabular}


FLA-ABS negative or Mitsuda negative/FLA-ABS positive. Nineteen $(5 \cdot 6 \%)$ out of these 340 individuals were Mitsuda negative but FLA-ABS positive. Seventeen had an antibody titre of $1: 40$ and 2 of $1: 160$.

\section{FLA-ABS TEST AMONG DOUBTFUL LEPROSY CASES}

The so-called doubtful cases were those who had skin lesion(s) or neural symptoms resembling leprosy when they first appeared. FLA-ABS tests had been carried out in 75 patients of this group and the results are shown in Table 6. The

Table 6. FLA-ABS test among doubtful leprosy cases grouping by clinical followed up results

\begin{tabular}{|c|c|c|c|c|c|c|c|c|c|c|}
\hline \multirow[b]{3}{*}{ Group } & \multirow{3}{*}{$\begin{array}{l}\text { No. } \\
\text { of } \\
\text { cases }\end{array}$} & \multirow{2}{*}{\multicolumn{2}{|c|}{ Positive }} & \multicolumn{7}{|c|}{ Distribution of antibody titre $\left(10 \times 4^{x}\right)$} \\
\hline & & & & & & $x=$ & & & Mean & \\
\hline & & No. & $\%$ & 0 & 1 & 2 & 3 & 4 & of $x$ & SD \\
\hline $\begin{array}{l}\text { Confirmed leprosy } \\
\text { during follow-up }\end{array}$ & 4 & 3 & $75 \cdot 0$ & 1 & 2 & 1 & 0 & 0 & $1 \cdot 00$ & $0 \cdot 82$ \\
\hline Requiring further follow-up & 13 & 7 & $53 \cdot 9$ & 6 & 4 & 2 & 1 & 0 & $0 \cdot 85$ & $0 \cdot 99$ \\
\hline $\begin{array}{l}\text { Ruled out leprosy } \\
\text { during follow-up }\end{array}$ & 58 & 9 & $15 \cdot 5$ & 49 & 7 & 2 & 0 & 0 & $0 \cdot 19$ & $0 \cdot 48$ \\
\hline Total & 75 & 19 & $25 \cdot 3$ & 56 & 13 & 5 & 1 & 0 & $0 \cdot 35$ & $0 \cdot 67$ \\
\hline
\end{tabular}

total positive rate was $25 \cdot 3 \%$. They had been followed up clinically in the Out-patient Department for at least 2 years before the test. About 1 year after the test, these patients were divided into three groups, as indicated below. Histopathological examinations were carried out on a sample of two-thirds of all patients studied. Group A with 4 cases whose lesion(s) gradually progressed with unequivocal leprosy, $3(75 \%)$ were FLA-ABS positive. Group B with 13 cases whose lesion(s) or symptoms had not significantly changed and still need further follow-up, $7(53.9 \%)$ were FLA-ABS positive. Group C with 58 cases, in whom the diagnosis of leprosy can be ruled out, $9(15.5 \%)$ were FLA-ABS positive. The differences in positive rate and mean antibody titre between group A vs B has no statistical significance but both groups were significantly higher than group $\mathrm{C}$. To analyse the outcome in groups $\mathrm{A}$ and $\mathrm{C}$, the $\chi^{2}$ test was used to check the correlation between the FLA-ABS test and the follow-up results (Table 7). The $\chi^{2}$ test was $7 \cdot 384$ and $P<0 \cdot 01$. Thus, the FLA-ABS positive doubtful cases were more prone to develop leprosy than the FLA-ABS negative cases. Among the 9 patients who were positive to FLA-ABS in group C, 7 cases came from leprosy endemic areas but the other 2 did not. The reasons for the positive tests in the latter 2 cases are unknown. 
Table 7. Correlation between FLA-ABS test and clinical follow up results of doubtful cases

\begin{tabular}{lccc}
\hline & \multicolumn{2}{c}{ Clinical follow up results } \\
FLA-ABS test & Confirmed leprosy & Ruled out leprosy & Total \\
\hline Positive & 3 & 9 & 12 \\
Negative & 1 & 49 & 50 \\
Total & 4 & 58 & 62 \\
\hline
\end{tabular}

CROSS-REACTION AND ADDITIONAL ABSORPTION OF POSITIVE FLA-ABS SERA

In order to show that a positive FLA-ABS test was not due to the retention of cross-reactive antibody to other mycobacteria in the sera after absorption with cardiolipin, lecithin, BCG and $M$. vaccae, 53 positive sera from non-leprosy individuals in Table 3 were tested for cross-reactivity with 6 other species of mycobacteria, i.e. M.tuberculosis, $M$. kansasii, M. marinum, $M$. smegmatis, $M$. phlei and $M$. avium. Only 5 demonstrated cross-reactivity with 1 or 2 species of mycobacteria, namely 3 to $M$. smegmatis, 2 to $M$. marinum and 1 to $M$. tuberculosis. These 5 sera were further absorbed with their corresponding mycobacteria. The cross-reaction disappeared in 4 sera but 1 was still positive to $M$. smegmatis though the antibody titre was reduced from $1: 160$ to $1: 40$. The antibody titre to $M$. leprae was not influenced by additional absorption with other mycobacteria. Ten positive FLA-ABS sera, 5 multibacillary patients, 4 household contacts and 1 professional contact, were tested by additional absorption with $M$. leprae, i.e. $0 \cdot 1 \mathrm{ml}$ serum absorbed by the routine procedures (resulting in a 10 -fold dilution) was mixed with $0.5 \mathrm{mg}$ freeze-dried $M$. leprae and incubated at $37^{\circ} \mathrm{C}$ for $30 \mathrm{~min}$. Before absorption with $M$. leprae, the antibody titre was $1: 160$ in $1,1: 640$ in 5 and $1: 2560$ in 4 . After absorption with $M$. leprae, the anti-M. leprae antibodies were completely removed.

\section{Discussion}

It is impossible to measure directly the sensitivity of FLA-ABS test for detecting subclinical leprosy infection, because at present no other specific reference systems are available. However, the results of the FLA-ABS test in leprosy patients and also in contacts can provide useful information. The present study revealed the following observations: (1) By the FLA-ABS test, anti-M. leprae antibodies have been detected in more than $90 \%$ of active leprosy patients. Even though the positive rate was least in TT patients, it still gave $73.9 \%$ (Table 1). (2) 


\section{4}

$J$ Baohong et al.

The positive rate and mean antibody titre correlated well with the bacterial load of the host or the activity of leprosy infection (Table 2). (3) Theoretically, subclinical infection should be greater in contacts and chances of subclinical infection should correlate with the infectivity of the index case, the intensity and the duration of exposure. The positive rate and mean antibody titre of the FLA-ABS test were much higher in household contacts of multibacillary patients than in those of paucibacillary patients

contacts with longer seniority than in those with shorter seniority (Table 4). From these findings, the FLA-ABS test seems to be quite sensitive for detecting subclinical leprosy infection. The following observations suggested that the FLA-ABS test is specific for leprosy infection: (1) All specimens from sputumpositive pulmonary tuberculosis patients and healthy non-contacts gave negative results (2) Only a small proportion of positive FLA-ABS sera from non-leprosy individuals showed cross-reaction to other mycobacteria and this can be differentiated by additional absorption with corresponding mycobacteria. (3) The reaction against $M$. leprae is completely removed when positive sera are further absorbed by $M$. leprae.

Since the FLA-ABS test is quite sensitive and specific for leprosy infection, the positive reaction in non-leprosy cases may be considered as an indication of subclinical infection. Because the positive rate in quiescent TT cases was much lower than in active cases, it would seem unlikely that the FLA-ABS test could detect past aborted infections and, therefore, the subclinical infection rate detected by this test might be an underestimate. Even though the figures in Table 3 demonstrated that the subclinical infection rate was at least 200 times higher than the cumulative prevalence rate of clinical infection at the same area, they are similar to those reported by Abe et al. ${ }^{3} \mathrm{~A}$ far greater proportion of the population are infected with $M$. leprae than develop clinical disease, indicating that the infections are aborted at the subclinical stage.

There was a correlation between a positive FLA-ABS test and the outcome in individuals with doubtful leprosy, in that they were more prone to develop leprosy later than those with a negative test. However, many doubtful cases came from endemic areas. It is difficult to differentiate the subclinical infection from very early leprosy by the FLA-ABS test. Hence, a positive FLA-ABS test is not a reliable indicator for the early diagnosis of leprosy.

The main purpose for detecting subclinical leprosy is to identify the individuals who have been infected and prone to develop into multibacillary leprosy. The FLA-ABS test alone can hardly fulfil this purpose because the great majority of leprosy patients are positive and the distribution of levels of antibody titres overlap between multibacillary and paucibacillary patients. It is possible to overcome this difficulty by including a lepromin test, because most multibacillary patients are in an anergic state, and therefore lepromin negative. A combination of a positive FLA-ABS test and a negative Mitsuda reaction indicates that the individual has been infected with $M$. leprae but cell-mediated immunity has not 
been induced. These individuals are more likely to develop multibacillary-type leprosy and most urgently need to be protected. Abe et al. used the frequency of individuals in this category as an index of susceptibility to leprosy. ${ }^{4}$ This index in the present study was $5 \cdot 6 \%$. Since the antibody titres of the FLA-ABS tests were rather low in all these individuals in our present study, we have followed them up carefully, including physical examination, lepromin and FLA-ABS tests. If this specific immunological status persists or the antibody titre tends to be increasing while the Mitsuda reaction is still negative, then prophylactic measures have been adopted.

\section{Acknowledgment}

This investigation received financial support from the Immunology of Leprosy (IMMLEP) and Chemotherapy of Leprosy (THELEP) components of the UNDP/World Bank/WHO Special Programme for Research and Training in Tropical Diseases. The authors express sincere thanks to Dr M Abe, National Institute for Leprosy Research, Tokyo, Japan for helping in standardization of FLA-ABS test and supplying part of reagents used in the test, and to Dr R J W Rees, National Institute for Medical Research, London, UK for his kind supply of $M$. leprae, and for his valuable suggestions and advice in preparing this manuscript.

\section{References}

1 Abe M, Izumi S, Saito T, Mathur, SK. Early serodiagnosis of leprosy by indirect immunofluorescence. Lepr India, 1976; 48: 272-6.

2 Abe M, Yoshino Y. Antigenic specificity of M. leprae by indirect immunofluorescence. Int $J$ Lepr, 1978; 46: 118.

${ }^{3}$ Abe M, Minagawa F, Yoshino Y, Ozawa T. Fluorescent leprosy antibody absorption (FLA-ABS) test for detecting subclinical infection with Mycobacterium leprae. Internat J Lepr, 1980; 48: 109-19.

4 Abe M, Ozawa T, Minagawa F, Yoshino Y. Subclinical infection in leprosy. Its detection and control by fluorescent leprosy antibody absorption (FLA-ABS) test. Lepr Rev, 1981; 52 (Suppl. 1): 263-73.

${ }^{5}$ Bharadwaj VP, Ramu G, Desikan KV. Fluorescent leprosy antibody absorption (FLA-ABS) test for early serodiagnosis of leprosy. Lepr India, 1981; 53: 518-24.

${ }^{6}$ Bharadwaj VP, Ramu G, Desikan KV. A preliminary report of subclinical infection in leprosy. Lepr India, 1982; 54: 220-7. 